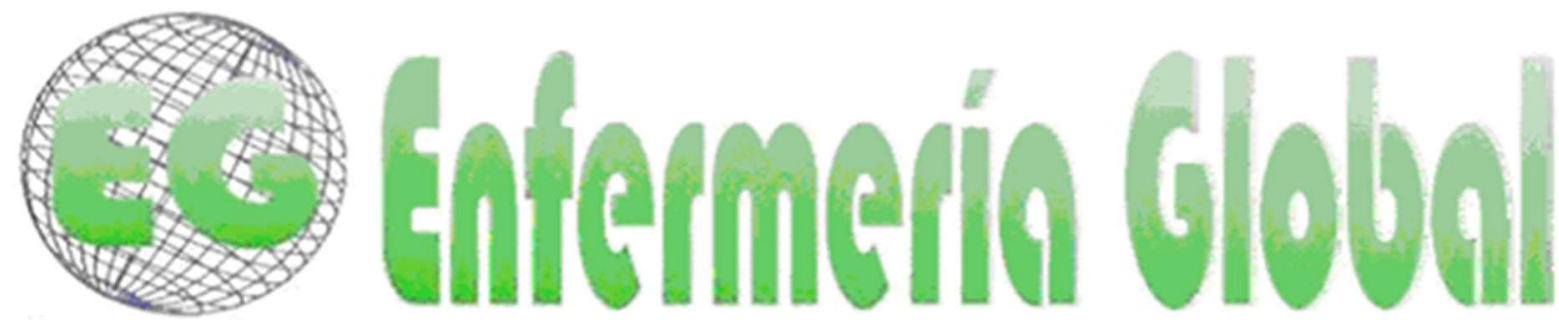

\title{
ORIGINALES
}

\section{Nivel de estrés y estrategias de afrontamiento utilizadas por estudiantes de la licenciatura en Enfermería}

Level of Stress and Coping Strategies used by Students of the Bachelor of Nursing

\begin{abstract}
Yenisel Carolina Valdez López ${ }^{1}$
Ricardo Antonio Marentes Patrón ${ }^{1}$

Sheida Eunice Correa Valenzuela ${ }^{2}$

Reyna Isabel Hernández Pedroza ${ }^{3}$

Isamar Daniela Enríquez Quintero²

María Olga Quintana Zavala ${ }^{3}$
\end{abstract}

\begin{abstract}
${ }^{1}$ Egresados de la Licenciatura en Enfermería Universidad de Sonora, Departamento de Ciencias de la Salud, Campus Cajeme. Ciudad Obregón, Sonora, México.

2 Docente de la Licenciatura de Enfermería, Universidad de Sonora, Departamento de Ciencias de la Salud, Campus Cajeme. Ciudad Obregón, Sonora, México. sheida.correa@unison.mx

3 Docente de la Licenciatura en Enfermería, Universidad de Sonora, Departamento de Enfermería, Campus Hermosillo. Hermosillo, Sonora, México.
\end{abstract}

\section{https://doi.org/10.6018/eglobal.441711}

Recibido: 2/09/2020

Aceptado: 3/03/2021

\section{RESUMEN:}

Objetivo: Determinar la relación entre el nivel de estrés y las estrategias de afrontamiento utilizadas por estudiantes de la Licenciatura en Enfermería en Instituciones de Educación Superior (IES) de Ciudad Obregón, Sonora.

Metodología: Estudio no experimental y correlacional; se empleó un muestreo probabilístico estratificado de 250 estudiantes, con edades entre los 18 y 46 años de edad. Los datos fueron recabados mediante un cuestionario de características sociodemográficas, el instrumento del nivel de estrés percibido y para medir afrontamiento el Brief COPE Inventory.

Resultados: El $74.4 \%$ de la muestra fueron mujeres y el $25.6 \%$ hombres, ambos géneros exhibieron nivel de estrés moderado (78\%), la estrategia de afrontamiento reportada con mayor frecuencia fue afrontamiento activo emocional. Se empleó la prueba no paramétrica chi-cuadrada de Pearson $\left(X^{2}\right)$ para probar la existencia de asociación entre el nivel de estrés y las estrategias de afrontamiento ( $p=$ $0.67)$. Con base a los resultados de $X^{2}(p=.067)$ se realizó un análisis adicional separando las categorías de cada una de las variables de estudio, se utilizó la prueba de Pearson con la cual se encontró correlación significativa entre el nivel de estrés bajo y la estrategia afrontamiento activo emocional, $(p=0.27)$ y entre nivel de estrés alto y estrategia de afrontamiento emocional por evitación $(p=-.01)$.

Conclusiones: Los niveles más altos de estrés lo manifiestan estudiantes que cursan solo materias teóricas. Se sugiere la implementación de intervenciones relacionadas al manejo adecuado del estrés.

Palabras claves: estrés, estrategias de afrontamiento, estudiantes del área de la salud (DeCS, BIREME). 


\begin{abstract}
:
Objective: Determine the relationship between the stress level and the coping strategies used by students of the Bachelor's Degree of Nursing in institutions of higher education of Ciudad Obregón, Sonora.

Methodology: Descriptive and correlational study; the population was comprised by 250 students whose age ranged from 18 and 46 years. Data was collected through a questionnaire of sociodemographic characteristics, the perceived stress level instrument, and the Brief COPE questionnaire.

Results: $74.4 \%$ of the sample was comprised by women and $25.6 \%$ by men. It was found that both genders had a moderate level of stress $(78 \%)$, in terms of the coping strategies most frequently used by the students, the emotional active coping was the one found. The correlation analysis was performed using Pearson's chi-square non-parametric test $\left(\mathrm{x}^{2}\right)$ to probe the existence of relationship between the stress level and coping strategies $(p=0.67)$. Based on the results of $x^{2}(p=0.067)$, an additional analysis was carried out separating the categories of each one of the variables of study; Pearson's test was used to find a significant correlation between low stress level and active emotional coping strategy $(p=0.27)$ and between high stress level and avoidance emotional coping strategy $(p=-0.01)$.

Conclusions: The highest levels of stress are manifested by students who study only theoretical subjects. It is suggested the implementation of interventions related to the adequate management of stress.
\end{abstract}

Keywords: Sress, coping strategies, students of the health area (DeCS, BIREME).

\title{
INTRODUCCIÓN
}

El estrés es un importante trastorno presente en el periodo de aprendizaje de una persona, con índices cada vez más alto en las sociedades del siglo XXI. La investigación sobre estrés y enfermedad humana se complica por el hecho de que el estrés es solo una condición que contribuye a la mayoría de los estados de enfermedad.

Se ha demostrado que los estudiantes de enfermería en las prácticas clínicas expresan una considerable percepción del estrés debido al trato directo con la vida humana ${ }^{(1)}$.

Desde un punto de vista fisiológico, "estrés" es cualquier situación que pone al cuerpo en estado de alerta. "Se trata de estados de malestar subjetivo, acompañados de alteraciones emocionales que, por lo general, interfieren con la actividad social y que aparecen en el periodo de adaptación a un cambio biográfico significativo o de un acontecimiento vital estresante" (2).

En el mundo "uno de cada cuatro individuos sufre de algún problema grave de estrés y en las ciudades, se estima que el 50 por ciento de las personas tienen algún problema de salud mental de este tipo" (3).

A fin de contextualizar, el estrés es considerado a nivel mundial como un problema de salud pública, debido a que afecta a toda la población, sin distinción. La Organización Mundial de la Salud (OMS), de acuerdo con Gutiérrez, menciona que a inicios del presente siglo las enfermedades provocadas por el estrés habrán superado a las enfermedades infecciosas y se estimó que, en el año 2010, en América Latina y el Caribe se tendrían más de 88 millones de personas con trastornos afectivos desencadenados por el estrés ${ }^{(4)}$.

Se debe tener en cuenta que los alumnos de enfermería por las características de la profesión "están en contacto con el sufrimiento, dolor, invalidez, e incluso la muerte", 
así como la interrelación con otros profesionales, están expuestos a mayor cantidad de estresores que otros grupos de estudiantes. Zryewsky y Davis ${ }^{(5)}$ describieron las principales fuentes de estrés de los estudiantes de enfermería y comprobaron que las áreas, académica y clínica generaban el $78.4 \%$ de los acontecimientos estresantes. El área social el $8 \%$ y el área personal el $13.6 \%$.

Betancourt, Hernández, Suárez y Arguello (6) demuestran que en los alumnos de ciencias médicas y biológicas el $39 \%$ de ellos obtiene un nivel de estrés muy elevado, $43 \%$ proyecta un nivel de moderado a ligeramente alto con tendencia a incrementarse en una situación que demande más de las ya vividas y un $18 \%$ indica un nivel de estrés de moderado a bajo. Se podría decir que más del $50 \%$ puede presentar una importante depresión o un problema mayor ocasionado por el estrés al que se someten.

Martín (7) señala que los índices de estrés en las poblaciones universitarias son notables, alcanzando mayores niveles en los primeros cursos de carrera que en los últimos y en los períodos inmediatamente anteriores a los exámenes. Debemos tener en cuenta que la exposición continua a situaciones estresantes puede provocar, por un lado, trastornos psicofisiológicos (por ejemplo, amenorrea, dolor de espalda, disfunciones sexuales, trastornos gastrointestinales, etc.) y mentales (por ejemplo, ansiedad, depresión, trastorno por estrés postraumático, etc.) y, por otro lado, aunque el estrés no provoque directamente las enfermedades, puede deteriorar el funcionamiento normal del organismo.

Se calcula que en México entre 30 y $40 \%$ de las personas sufren estrés: "somos el segundo país a nivel mundial con el índice más alto en incidencia de este problema, después de China", reveló Alfredo Whley Sánchez, médico adscrito de la consulta externa del Instituto Nacional de Psiquiatría Ramón de la Fuente Múñiz (INPRF) ${ }^{(8)}$.

Actualmente se desconocen estudios locales sobre el estrés universitario y sobre todo en estudiantes de enfermería quienes tienen entre sus responsabilidades académicas el cuidado de personas reales en sus prácticas clínicas, eso los hace propensos a presentar niveles altos de estrés. Es importante para las instituciones conocer las estrategias de afrontamiento utilizadas por los estudiantes para realizar intervenciones de enfermería convenientes que contribuyan al desarrollo integral de los alumnos.

\section{OBJETIVOS}

- Determinar la relación entre el nivel de estrés y las estrategias de afrontamiento utilizadas por estudiantes de la Licenciatura en Enfermería en Instituciones de Educación Superior (IES) de Ciudad Obregón, Sonora.

- Describir las características sociodemográficas de estudiantes de la Licenciatura en Enfermería de Instituciones de Educación Superior (IES) de Cd. Obregón, Sonora.

- Determinar el nivel de estrés de estudiantes de la Licenciatura de Enfermería de IES.

- Determinar las estrategias de afrontamiento utilizadas por los estudiantes de la Licenciatura de Enfermería.

- Relacionar las estrategias de afrontamiento utilizadas por estudiantes de la Licenciatura en Enfermería de las distintas Instituciones de Educación Superior (IES) según el nivel de estrés percibido. 


\section{METODOLOGÍA}

\section{Diseño del estudio}

El diseño del estudio fue de tipo correlacional con abordaje cuantitativo y de corte transversal; tiene como objetivo medir el grado de relación que existe entre dos o más conceptos o variables. Se buscó asociar la relación que existía entre el nivel de estrés y las estrategias de afrontamiento.

\section{Población, Muestreo y Muestra}

Se consideró a los estudiantes de enfermería de las distintas Instituciones de Educación Superior de Ciudad Obregón, Sonora. La población total para el presente estudio fue de 750 estudiantes de Enfermería; UNISON (234 alumnos), Escuela Iberoamericana (146 alumnos) y el Instituto de Formación y Capacitación de Profesionales de Enfermería (IFCPE, 335 alumnos). Mediante la fórmula para poblaciones finitas se calculó una muestra de $n=250$ estudiantes y para la selección de éstos se utilizó el método de muestreo probabilístico estratificado. Primeramente, se estratificó por universidad y posteriormente por semestre.

Criterios de inclusión: Estudiantes de la Licenciatura de Enfermería de las distintas Instituciones de Educación Superior de Cd. Obregón, Sonora.

Criterios de exclusión: Aquellos alumnos que padezcan una enfermedad que pueda alterar los resultados del estudio como por ejemplo ansiedad, depresión o bien que estén en tratamiento médico/psicológico/psiquiátrico en curso o hace menos de 6 meses.

Criterios de eliminación. Estudiantes que dejaron irresueltos los instrumentos de evaluación, estudiantes sin deseo de participar en el estudio.

\section{Procedimiento de recolección de la información}

Para la recolección de la información se solicita la autorización del Comité de Ética en Investigación (CEI) del Departamento de Enfermería de la Universidad de Sonora, Campus Hermosillo. Una vez obtenida la resolución del CIE, se procedió a visitar a las distintas Instituciones de Educación Superior para solicitar permiso de manera verbal y por oficios para poder aplicar los instrumentos de evaluación en la población estudiantil.

Se solicitó información a las mismas acerca de la cantidad de estudiantes y la cantidad de alumnos por grupo, para después obtener el número de instrumentos que se aplicarían por cada IES y por grupo.

La recolección de los datos se llevó a cabo por los investigadores principales a través de la visita a las IES (UNISON, IFCPE, IBEROAMERICANA) con el fin de unificar criterios y evitar sesgos durante la recolección de datos. Para la recolección de la información se aplicaron tres instrumentos, el primero para conocer datos sociodemográficos de los estudiantes diseñado por los responsables de la investigación; el segundo instrumento fue el que determinó el nivel de estrés, y el 
último instrumento con el fin de medir la variable de estrategias de afrontamiento. Al terminar con la aplicación de los instrumentos se agradecía por la participación en el estudio.

\section{Instrumentos y mediciones}

Para la recolección de datos sociodemográficos se elaboró un instrumento que contenía los datos necesarios para la ejecución de la investigación, en dicho instrumento se preguntó lo siguiente: institución a la que pertenece el alumno, edad, sexo, semestre, ocupación, número de hijos.

Para evaluar el nivel de estrés académico se utilizó la Escala de Estrés Percibido (Perceived Stress Scale, PSS-14) el cual es un auto informe que evalúa el nivel de estrés percibido durante el último mes, consta de 14 ítems con un formato de respuesta de una escala de cinco puntos $(0=$ nunca, $4=$ muy a menudo). La puntuación total de la PSS se obtiene invirtiendo las puntuaciones de los ítems 4, 5, 6, $7,9,10$ y 13 (en el sentido siguiente: $0=4,1=3,2=2,3=1$ y $4=0$ ) y sumando entonces los 14 ítems. La puntuación directa obtenida indica que a una mayor puntuación corresponde un mayor nivel de estrés percibido ${ }^{(9)}$.

Para identificar las estrategias de afrontamiento utilizadas por los estudiantes de las diversas instituciones educativas se utilizó el cuestionario Brief COPE Inventory (versión breve de Carver, COPE-28), el cual contiene 28 ítems de tipo Likert de cuatro alternativas de respuesta cuya valoración va desde 1 (No he estado haciendo esto en absoluto) hasta 4 (he estado haciendo esto mucho). Esta escala se centra en evaluar las estrategias de afrontamiento que manejan las personas ante el estrés. Los 28 ítems se agrupan de 2 en 2 dando como resultado 14 estrategias de afrontamiento contenidas en dos dimensiones una denominada afrontamiento activo y el otro afrontamiento pasivo. Todos los ítems son positivos. A mayor puntuación en cada modo de afrontamiento mayor utilización de esa estrategia. Las 14 subescalas se agruparán en 3 categorías de afrontamiento sumando los elementos en puntuaciones más altas que indicarán mayor intensidad de uso de la estrategia. Los ítems fueron factorizados en: centrado en el problema como afrontamiento activo, planificación, instrumental de apoyo, y religiosidad $(\alpha=0.80)$; afrontamiento activo emocional en forma de ventilación, replanteamiento positivo, humor, aceptación, y apoyo emocional $(\alpha=0.81)$, y afrontamiento emocional por evitación en forma de auto distracción, negación, desconexión conductual, auto culpa y uso de sustancias $(\alpha=0.88){ }^{(10)}$.

\section{Consideraciones éticas y legales}

Antes de la aplicación de los instrumentos, el protocolo de investigación fue aprobado por el Comité de Ética en Investigación del Departamento de Enfermería de la Universidad de Sonora, con registro CEI-ENFERMERIA-E-77/2018.

El estudio cumplió con los aspectos éticos que garantizan el respeto a la dignidad y protección de los derechos y el bienestar de los alumnos. En el desarrollo de este se consideró el Reglamento de la Ley General de Salud (11) en materia de investigación para la Salud; solicitando el consentimiento informado verbal y por escrito a sujetos de estudio para garantizar una participación voluntaria y libre. El estudio se clasificó como investigación sin riesgo (artículo 17). También se siguieron los principios del 
convenio sobre los derechos del hombre y la biomedicina, conocido como Convenio de Oviedo ${ }^{(12) .}$

Previo a la firma del consentimiento informado los participantes fueron avisados del beneficio de su colaboración, tanto para ellos como para otros estudiantes y profesionales de enfermería. Se les explicó que la información que proporcionarían sería manejada confidencial y únicamente siguiendo los fines de la investigación. Además, se tuvo cuidado extremo en el manejo de los instrumentos, se encontraron resguardados bajo llave y cuando fue oportuno, la información se incinerada por respeto a las unidades de investigación.

\section{Análisis estadístico de los datos}

Los datos se analizaron a través software estadístico SPSS versión 23; se utilizó estadística descriptiva para el análisis de variables sociodemográficas y estadística no paramétrica para la correlación de variables, a través de la prueba Chi cuadrada $\left(X^{2}\right)$ y correlación de Pearson.

\section{RESULTADOS}

El total del tamaño de la muestra fue de 250 estudiantes de los cuales el $32.8 \%$ realiza sus estudios en la Universidad de Sonora (UNISON), $46.8 \%$ lo hace en el Instituto de Formación y Capacitación de Profesionales en Enfermería (IFCPE) y el $20.4 \%$ restante en la escuela de Enfermería Iberoamericana (IBERO).

En la Licenciatura en Enfermería las mujeres representan casi tres cuartas partes de la totalidad de los estudiantes $(74.4 \%)$ mientras que los hombres representan solo la cuarta parte restante $(25.6 \%)$. La media de edad de los participantes es de 21.76 años $(D E= \pm 3.58)$. La mayoría de los estudiantes son jóvenes, el $62.8 \%$ se encuentra en un rango de edad entre 18 y 21 años, $25.2 \%$ se encuentra en el rango de entre los 22 y 25 años y el $12 \%$ se encuentra distribuido entre los 26 y 46 años; siendo 18 años la edad mínima y 46 años la máxima.

La mayoría de los estudiantes se encuentran en estado civil de soltería con $91.6 \%$, el $6.8 \%$ se encuentran casados y solo el $1.6 \%$ están separados o divorciados. El $86.8 \%$ de los estudiantes no tienen hijos. En cuanto a la actividad laboral, el $68.8 \%$ de los alumnos no trabajan, mientras que el $31.2 \%$ sí realiza actividades laborales.

La mayoría de los alumnos se encuentran cursando el segundo y cuarto semestre, ambos con $31.2 \%$; mientras que el $18.8 \%$ cursa sexto semestre y el $18.8 \%$ restante cursa el octavo semestre.

\section{Variables de estudio}

En la tabla 1 se muestra que el $21.2 \%$ de los estudiantes mostraron un nivel de estrés bajo, el $78 \%$ tuvo nivel de estrés moderado y $0.8 \%$ presentó un nivel de estrés alto. 
Tabla 1. Nivel de estrés en estudiantes de la Licenciatura en Enfermería por institución educativa, Cd. Obregón, Sonora, 2018.

\begin{tabular}{|c|c|c|c|c|c|c|}
\hline \multirow[t]{2}{*}{ Variable / IES } & \multicolumn{2}{|c|}{ UNISON } & \multicolumn{2}{|c|}{ IFCPE } & \multicolumn{2}{|c|}{ IBERO } \\
\hline & f & $\%$ & f & $\%$ & f & $\%$ \\
\hline \multicolumn{7}{|l|}{ Nivel de Estrés } \\
\hline Bajo & 15 & 6 & 32 & 12.8 & 6 & 2.4 \\
\hline Moderado & 65 & 26 & 85 & 34 & 45 & 18 \\
\hline Alto & 2 & 0.8 & 0 & 0 & 0 & 0 \\
\hline Total & 82 & 32.8 & 117 & 46.8 & 51 & 20.4 \\
\hline
\end{tabular}

En la tabla 2 se presentan las estrategias de afrontamiento ante el estrés más utilizadas por los estudiantes de la Licenciatura en Enfermería de las diferentes instituciones de educación superior. Se encontró que utilizan mayormente el afrontamiento activo emocional (60.4\%), dentro del cual se incluyen acciones como: la descarga emocional, humor, aceptación, búsqueda de apoyo emocional y reformulación positiva. En segundo lugar, se encuentra el afrontamiento centrado en el problema (37.2\%), que incluye: el afrontamiento activo, de planeación, de apoyo con algún instrumento y la religión. Sólo el $2.4 \%$ utiliza el afrontamiento emocional por evitación, que se caracteriza por: autodistracción, negación, renuncia, auto culpa y uso de sustancias.

Tabla 2. Estrategias de afrontamiento utilizadas por los estudiantes de la Licenciatura en Enfermería por institución educativa, Cd. Obregón, Sonora, 2018.

\begin{tabular}{|c|c|c|c|c|c|c|}
\hline \multirow[t]{2}{*}{ Variable / IES } & \multicolumn{2}{|c|}{ UNISON } & \multicolumn{2}{|c|}{ IFCPE } & \multicolumn{2}{|c|}{ IBERO } \\
\hline & $f$ & $\%$ & $f$ & $\%$ & $f$ & $\%$ \\
\hline \multicolumn{7}{|l|}{$\begin{array}{l}\text { Estrategias de } \\
\text { Afrontamiento }\end{array}$} \\
\hline $\begin{array}{l}\text { Afrontamiento centrado en } \\
\text { el problema }\end{array}$ & 27 & 10.8 & 44 & 17.6 & 22 & 8.8 \\
\hline $\begin{array}{l}\text { Afrontamiento activo } \\
\text { emocional }\end{array}$ & 53 & 21.2 & 70 & 28 & 28 & 11.2 \\
\hline $\begin{array}{l}\text { Afrontamiento emocional } \\
\text { por evitación }\end{array}$ & 2 & 0.8 & 3 & 1.2 & 1 & 0.4 \\
\hline Total & 82 & 32.8 & 124 & 46.8 & 51 & 20.4 \\
\hline
\end{tabular}

La tabla 3 muestra el análisis entre el nivel de estrés y las estrategias de afrontamiento, se puede observar que porcentualmente el nivel de estrés bajo y moderado se asocia con el afrontamiento activo emocional. 
Tabla 3. Nivel de estrés y estrategias de afrontamiento de estudiantes de la Licenciatura en Enfermería, Cd. Obregón, Sonora, 2018.

\begin{tabular}{|l|c|c|c|}
\hline Variables & $\begin{array}{c}\text { Afrontamiento } \\
\text { centrado en el } \\
\text { problema } \\
\mathbf{f}\end{array}$ & $\begin{array}{c}\text { Afrontamiento } \\
\text { activo emocional } \\
\mathbf{f}\end{array}$ & $\begin{array}{c}\text { Afrontamiento } \\
\text { emocional por } \\
\text { evitación } \\
\mathbf{f}\end{array}$ \\
\hline Nivel de estrés & 14 & & \\
\hline Bajo & 77 & 39 & 0 \\
\hline Moderado & 2 & 112 & 6 \\
\hline Alto & 93 & 0 & 6 \\
\hline Total & 151 & $n$ \\
\hline Fuente: Cuestionario Brief COPE Inventory, COPE1997; Perceived Stress Scale, 2001.
\end{tabular}
250

\section{Análisis correlacional}

Al utilizar la prueba no paramétrica de chi-cuadrado de Pearson $\left(x^{2}\right)$, no se encontró una asociación estadísticamente significativa entre la variable nivel de estrés y las estrategias de afrontamiento (tabla 4).

Tabla 4. Prueba de Chi-cuadrado en las variables nivel de estrés y estrategias de afrontamiento.

\begin{tabular}{|l|c|c|c|}
\hline & \multicolumn{3}{|c|}{ Pruebas de chi-cuadrado } \\
\hline & Valor & gl & $\begin{array}{c}\text { Significación } \\
\text { asintótica } \\
\text { (bilateral) }\end{array}$ \\
\hline Chi-cuadrado de Pearson & $8.761^{\mathrm{a}}$ & 4 & .067 \\
\hline N de casos válidos & 250 & & \\
\hline
\end{tabular}

Fuente: Cuestionario Brief COPE Inventory, 1997; Perceived Stress Scale, $2001 . \quad \mathrm{N}=250$

Con base a los resultados de $x^{2}(p=.067)$ se realizó un análisis adicional separando las categorías de cada una de las variables de estudio, se utilizó la prueba de Pearson con la cual se confirman los resultados anteriores, al obtener valores de superiores a $(p=0.5)$, se rechaza la hipótesis de investigación, sin embargo se evidenció la correlación significativa entre el nivel de estrés bajo y la estrategia utilizada en cuanto al afrontamiento activo emocional $(p=.02)$, además de una correlación negativa entre nivel de estrés alto y afrontamiento emocional por evitación $(p=-.01)$.

Tabla 5. Prueba de correlación de Pearson en las variables nivel de estrés y estrategias de afrontamiento.

\begin{tabular}{|c|c|c|c|c|c|c|}
\hline \multicolumn{7}{|c|}{ Pruebas de correlación de Pearson } \\
\hline & \multicolumn{2}{|c|}{$\begin{array}{c}\text { Afrontamiento } \\
\text { centrado en el } \\
\text { problema }\end{array}$} & \multicolumn{2}{|c|}{$\begin{array}{c}\text { Afrontamiento activo } \\
\text { emocional }\end{array}$} & \multicolumn{2}{|c|}{$\begin{array}{c}\text { Afrontamiento } \\
\text { emocional por } \\
\text { evitación }\end{array}$} \\
\hline $\begin{array}{l}\text { Nivel de } \\
\text { Estrés }\end{array}$ & $\mathbf{r}$ & $p$ & $\mathbf{r}$ & $\mathbf{p}$ & $\mathbf{r}$ & $\mathbf{p}$ \\
\hline Bajo & -.116 & .068 & .140 & .027 & -.081 & .200 \\
\hline Moderado & .089 & .160 & -.114 & .072 & .083 & .189 \\
\hline
\end{tabular}




\begin{tabular}{l|c|c|c|c|c|r} 
Alto & .117 & .065 & -.111 & .080 & -.014 & .825 \\
\hline Fuente: Cuestionario Brief COPE Inventory, 1997; Perceived Stress Scale, 2001. & $\mathrm{n}=250$.
\end{tabular}

\section{DISCUSIÓN}

La muestra estuvo conformada por 250 estudiantes universitarios de los cuales el $32.8 \%$ (82 alumnos) cursan la Licenciatura de Enfermería en la Universidad de Sonora (UNISON), 46.8\% (117 alumnos) en el Instituto de Formación y Capacitación de Profesionales en Enfermería (IFCPE) y el 20.4\% (51 alumnos) en la Universidad Iberoamericana (IBERO).

El perfil sociodemográfico de los alumnos refleja que la mayoría de los estudiantes de enfermería pertenece al género femenino $74.4 \%$, solo el $25.6 \%$ representa al género masculino; con una edad media de 21.76 años ( $D E= \pm 3.58$ ). Lo que se asemeja a los datos proporcionados por el Instituto Nacional de Estadística y Geografía (INEGI) y su Encuesta Nacional de Ocupación y Empleo (ENOE) del segundo trimestre del 2015, en México la población mayor de 15 años ocupada como enfermera o enfermero es de 475,295 personas, de las cuales $85 \%$ son mujeres y $15 \%$ hombres ${ }^{(13)}$.

El conjunto de características que conforman el perfil de los alumnos nos muestra una población joven, un estudio realizado por De Valle \& col. (14) asemeja los resultados obtenidos ya que su estudio decretó que la media de edad fue de 20 años $(D E=4.3)$; con predominio del género femenino $(73 \%)$ como es característico de la carrera de enfermería.

Los datos que se obtuvieron en esta investigación muestran que tanto el género masculino como el femenino representaron un nivel de estrés moderado $78 \%$ (195 alumnos). Datos similares arroja la investigación realizada por Rivas Acuña y col. ${ }^{(15)}$ llamada Frecuencia e Intensidad del Estrés en Estudiantes de Licenciatura en Enfermería de la DACS, la frecuencia e intensidad del estrés académico en los estudiantes de la Lic. Enfermería de la UJAT-DACS en general, se presentó poco frecuente con un $46 \%$, pero con una intensidad moderada en el $55 \%$.

Respecto a la variable de estrategias de Afrontamiento los estudiantes de Enfermería de las distintas (IES) para confrontar el estrés con mayor frecuencia utilizan el Afrontamiento activo emocional $60.4 \%$, el $37.2 \%$ Afrontamiento centrado en el problema y solo el $2.4 \%$ manejan el Afrontamiento emocional por evitación.

Según diversos estudios, quienes utilizan estrategias de afrontamiento centradas en el problema y estrategias emocionales más funcionales, se adaptan mejor, pudiendo obtener mayor éxito en lo académico, laboral, etc. (16) al enfrentar los eventos de manera más activa.

Moreano ${ }^{(17)}$, en una muestra de 145 estudiantes, encontró una mayor preferencia por el uso de estilos centrados en el problema, como esforzarse y tener éxito, al igual que Torrejón ${ }^{(18)}$.

En un estudio con 123 estudiantes de Psicología (19), encontraron que las estrategias de afrontamiento usadas con más frecuencia eran la reinterpretación positiva y la búsqueda de soporte social; y los estilos de afrontamiento más usados, fueron mixtos 
y básicamente centrados en el problema y en la emoción. Resultados similares obtuvo Chau (20), encontrando como estilo de afrontamiento más usado el centrado en el problema, seguido por la emoción y en último lugar la evitación.

Para los estudiantes de enfermería hay muchos estresores. Zryewskyj y Davis (5) describieron las principales fuentes de estrés de los estudiantes de enfermería; comprobaron que las áreas académicas y clínicas generaban el $78.4 \%$ de los acontecimientos estresantes.

En este estudio se demostró que aquellos que no cursan práctica clínica experimentan mayor nivel de estrés con $31.2 \%$ y los alumnos que están en las prácticas profesionales con un $18.2 \%$.

Muchos investigadores han estudiado el estrés y las situaciones estresantes de los alumnos de enfermería durante sus prácticas clínicas, destacando el periodo inicial de estas prácticas como el más estresante respecto a otros periodos.

Al estudiar la relación entre el nivel de estrés y las estrategias de afrontamiento no se encontró una diferencia significativa al obtener un valor de $p$ de 0.67 . Lo que indica que no hay relación entre éstas por lo que la hipótesis de estudio se rechaza. Este resultado concuerda con un estudio denominado estrés y técnicas de afrontamiento en estudiantes universitarios de Quito (17) donde el resultado que se obtuvo fue de $p=0.1272$ lo que indica que es un valor mayor al nivel de significancia.

Se encontró evidencia de correlación significativa entre el nivel de estrés bajo y la estrategia afrontamiento activo emocional $(p=0.27)$ y entre nivel de estrés alto y estrategia de afrontamiento emocional por evitación $(p=-.01)$.

Se ha estudiado el estrés y las situaciones estresantes de los alumnos de enfermería durante sus prácticas clínicas, destacando el periodo inicial de estas prácticas como el más estresante respecto a otros periodos ${ }^{(1,3,5)}$.

\section{CONCLUSIONES}

Las características sociodemográficas de la población en estudio fueron: género, predominante el femenino, edad media de 21.76 años, solteros, sin hijos, la mayoría de la población en estudio no trabaja.

Del total de la muestra la mayoría presenta niveles de estrés moderado; quienes apuntaron mayor nivel de estrés son: de género femenino, estudiantes solteros, sin hijos, que no trabajan, alumnos inscritos en los primeros semestres de la universidad y quienes aún no están suscritos en ninguna práctica clínica. Como se mencionó con anterioridad, los niveles más altos de estrés lo manifiestan estudiantes del primer curso y se alude a que los alumnos de grados más elevados han desarrollado habilidades para contrarrestar las situaciones estresantes.

En lo que se refiere a las estrategias de afrontamiento, los estudiantes mostraron una mayor tendencia hacia el afrontamiento activo emocional, el cual se refiere a los pasos activos para tratar de cambiar las situaciones o aminorar sus efectos. En segundo lugar, el afrontamiento centrado en el problema, que implica el manejo de las demandas internas o ambientales que suponen una amenaza y descompensan la 
relación entre la persona y su entorno. Finalmente, el afrontamiento emocional por evitación donde el universitario utiliza estrategias centradas en la evasión, la distracción, toma distancia del evento estresante, o se vuelca en otra actividad para no pensar en el problema.

En la población estudiada no se encontró una asociación significativa entre las variables nivel de estrés y estrategias de afrontamiento.

\section{REFERENCIAS}

(1) López-Medina IM, Sánchez-Criado V. Percepción del estrés en estudiantes de enfermería en las prácticas clínicas. Enfermería Clínica. 2005; 15(6): 307-313.

(2) Cuatrecasas G. Estrés y dolor crónico: una perspectiva endocrinológica. Reumatol Clin. (2009); 5(S2):12-14.

(3) Caldera JF, Pulido BE, Martínez MG. Niveles de estrés y rendimiento académico en estudiantes de la carrera de Psicología del Centro Universitario de Los Altos. Revista de Educación y Desarrollo. 2007; 7:77-82.

(4) Organización Mundial de la Salud, OMS: La inversión en el tratamiento de la depresión y la ansiedad. [Internet]. [Consultado 08 may 2018]. Disponible en: https://www.who.int/mediacentre/news/releases/2016/depression-anxiety-

treatement/es/

(5) Zryewskyj T, Davis L. Sources of stress in third year baccalaureate nursing students. AARN News Lett. 1987 Mar;43(3):24-5. PMID: 3646825.

(6) Betancourt, A., Hernández, A. y Suárez, J. El Estrés. Trabajo de investigación. Facultad de Ciencias Médicas. Universidad Michoacana de San Nicolás de Hidalgo; 2006.

(7) Martín, I. Estrés académico en estudiantes universitarios. Apuntes de Psicología Colegio Oficial de Psicología. 2007; 25(1): 87-99.

(8) Rosenzweig \& Leiman. Psicología Fisiológica. McGraw-Hill; 1992.

(9) Moral J, Cázares F. Validación de la Escala de Estrés Percibido (PSS-14) en la población de dentistas colegiados de Monterrey. Ansiedad y Estrés. 2014; 20(2-3): 193-209.

(10) Schnider KR, Elhai JD, Gray MJ. Coping style use predicts posttraumatic stress and complicated grief symptom severity among college students reporting a traumatic loss. Journal of Counseling Psychology. 2007; 54(3): 344-350. https://doi.org/10.1037/0022-0167.54.3.344

(11) REGLAMENTO de la Ley General de Salud en Materia de Investigación para la Salud. Disponible en: http://www.salud.gob.mx/unidades/cdi/nom/compi/rlgsmis.html

(12) Convenio de Oviedo. Disponible en: http://www.bioeticanet.info/documentos/Oviedo1997.pdf

(13) INEGI. Instituto Nacional de Estadística y Geografía. Encuesta Intercensal (2015). Disponible en

http://internet.contenidos.inegi.org.mx/contenidos/productos/prod_serv/contenidos/esp anol/bvinegi/productos/nueva_estruc/promo/eic_2015_presentacion.pdf

(14) Di-Colloredo C, Aparicio D, Moreno J. Descripción de los estilos de afrontamiento en hombres y mujeres ante la situación de desplazamiento. Psychologia. Avances de la disciplina. 2007; 1(2): 125-156.

(15) Rivas V, Jiménez C, Méndez H, Cruz M, Magaña M, Victorino A. Frecuencia e Intensidad del Estrés en Estudiantes de Licenciatura en Enfermería de la DACS. Horizonte Sanitario. 2014; 13(1): 162-169. 
(16) Cornejo M, Lucero M. Preocupaciones vitales en estudiantes universitarios relacionados con bienestar psicológico y modalidades de afrontamiento. Fundamentos en Humanidades. 2005; 6(12): 143-153.

(17) Moreano, L. Estilos de personalidad y estrategias de afrontamiento en adolescentes universitarios. Tesis para optar por el Título de Licenciada en Psicología con mención en Psicología Clínica. Lima-Perú. 2006.

(18) Torrejón C. Ansiedad y afrontamiento en universitarios migrantes. Tesis para optar por el título de Licenciada en Psicología con mención en Psicología Clínica. PUCP, Lima-Perú. 2011.

(19) Caldera JF, Pulido BE, Martínez MG. Niveles de estrés y rendimiento académico en estudiantes de la carrera de Psicología del Centro Universitario de Los Altos [Versión electrónica]. Revista de Educación y Desarrollo. 2007; 7: 77-82.

(20) Chang, E.C. Optimismo disposicional y evaluación primaria y secundaria de un factor estresante: Controlar las influencias y las relaciones de confusión con el afrontamiento y el ajuste psicológico y físico. Revista de Personalidad y Psicología Social. 1998; 74: 1109-1120. 